\title{
Irreversibility and Restoration in Natural Resource Development
}

\author{
Jinhua Zhao David Zilberman ${ }^{1}$
}

September 15, 1998

${ }^{1}$ The authors are an assistant professor at the Department of Economics, Iowa State University, and a Professor at the Department of Agricultural and Resource Economics, University of California at Berkeley. Please address all correspondences to the first author. We would like to thank Anthony Fisher, Michael Hanemann, Hayne Leland, Brian Wright, and an anonymous referee for their helpful comments. The usual disclaimer applies. 


\begin{abstract}
We extend Real Option Theory to evaluate natural resource development projects that may bring negative net benefits and require costly restoration. Based on a new concept, irreversibility cost, we show that the degree of irreversibility becomes an endogenous choice, rather than an exogenously given economic constraint. The fixed cost of restoration has continuous impacts, over and above the widely recognized fixed effects, on the level of development and restoration (and the marginal $q$ ). Further, the value of the project may not necessarily be convex in the underlying random variable, and discounting may in fact encourage the pattern of developing now and restoring later.
\end{abstract}


The standard tool of project evaluation, the Net Present Value criterion, seems applicable to natural resource projects, such as a hydroelectric dam or clear-cutting land for agriculture. But as Arrow and Fisher (1974) argues, resource project evaluation has to account for restoration difficulties in case of project failures. This feature has generated a rich literature on investment decisions under uncertainty and irreversibility, or Real Option Theory (ROT). ${ }^{1}$ The major argument of ROT is that facing irreversibility and uncertainty, it may pay to delay the investment and wait for information to avoid the downside risk. Thus, conventional project evaluation leads to too early or too much investment.

The assumption of absolute irreversibility in ROT is in most cases too strong, given resource restoration efforts currently going on around the world. ${ }^{2}$ Fish population reduced by a dam may be restored by decreasing the capacity of or even destroying the dam. ${ }^{3}$ Clear cutting forest may endanger valuable species, and restoration involves reforestation to preserve these species.

Most of the ROT literature ${ }^{4}$ focus on the timing of investment and do not allow variable project size. We call this 0-1 approach since the decision for the current period is either full (the "1") or no development (the "0"). The major conclusion of the 0-1 model is that increased uncertainty tends to delay the investment. Pindyck (1988) considers variable project size but does not allow costly restoration. He concludes that increased uncertainty reduces the level of investment.

This paper aims to extend ROT to allow for restoration of natural resource projects that may result in negative environmental benefits. Irreversibility is not assumed, instead economic optimization determines the extent of restoration and whether zero restoration (irreversibility) is

\footnotetext{
${ }^{1}$ see Arrow and Fisher (1974), Henry (1974), Dixit and Pindyck (1994), Epstein (1980), Freixas and Laffont (1984), Ulph and Ulph (1997), and Kolstad (1996).

${ }^{2}$ There are efforts to restore the Everglades in Florida, the San Francisco Bay Delta in California, the Rhon River in France, the Pearl River in China, and the Hula Lake in Isreal, among others.

${ }^{3}$ Destroying the Elwha dam in the state of Washington has been studied seriously as a way of restoring the ecosystem and fisheries in the Elwha River area.

${ }^{4}$ They include Arrow and Fisher (1974), Henry (1974), Capozza and Li (1994), McDonald and Siegel (1986), Majd and Pindyck (1987), Dixit and Pindyck (1994) and a series of papers by Pindyck.
} 
optimal. We go beyond the 0-1 approach and assume that the size of the project is endogenously chosen in all periods. Thus, resource management is modeled as a closed-loop stochastic dynamic optimization problem that determines the extent of both investments and restoration, and the probability and degree of irreversibility.

We introduce a new concept, called irreversibility cost (IC), which measures the expected loss due to costly restoration and irreversibility. IC is monotonically related to the Arrow-Fisher-Henry option value $(\mathrm{OV})$ in problems of fixed project size and absolute irreversibility, but is applicable to more general problems of variable project size and costly restoration. IC highlights the implications of negative net benefit of development, and the adjustment in the optimal project scale it entails.

The concept of restoration emphasized here is related to the disinvestment concept in Abel and Eberly (1994). In their case, disinvestment is undertaken when selling the equipment is more profitable than continuing the operation. Our model is different because in resource development, the reason for restoration is negative environmental impact. We will obtain some counterintuitive results, showing that the value of resource projects may decline with future uncertainty, and that increased discount rate may encourage investment.

For natural resource projects, there is an important distinction between technical and economic irreversibility. The former happens when there does not exist a technology that can mitigate the negative impacts of the development. Extinction of endangered species due to resource development falls into this category. The original work of Arrow and Fisher (1974) and some of its applications (such as Fisher, Krutilla and Cicchetti (1972)) seem to assume technical irreversibility. Economic irreversibility arises when it is not optimal to restore the development even though technologies exist for doing so. For example, Caballero (1991) considered irreversibility as corresponding to infinite marginal cost for negative adjustments of the project size. Our framework applies to technical irreversibility but we focus on economic irreversibility. 
The paper is organized as follows. In Section 1, we assume irreversibility to illustrate an analytical framework based on a new concept, irreversibility cost. We compare irreversibility cost with the option value defined in Arrow and Fisher (1974) and Henry (1974). We replicate some of the standard results of ROT using the new framework, and show that in our model higher discount rate promotes development. Section 2 considers costly restoration and shows that the degree of irreversibility is an endogenous choice. It also illustrates that the fixed cost of restoration has continuous impacts on levels of development and restoration. Section 3 concludes the paper and discusses implications of the results and possible future research. The appendices include model details and proof of the theorems.

\section{Basic Model and Irreversibility Cost}

We consider a risk neutral decision maker facing a net benefit function $B^{t}\left(K_{t}, w_{t}\right)$, where $K_{t}=$ $\sum_{s=1}^{t} I_{s}$ is the total capacity of the project, $I_{t}$ is the level of development (or restoration if $I_{t}$ is negative), and $w_{t}$ is the random variable in period $t$, for $t=1,2 .{ }^{5}$ We assume that (A1) $B^{t}\left(K_{t}, w_{t}\right)$ is concave in $K_{t}$, (A2) $B_{K_{t} w_{t}}^{t}>0$, (A3) $B_{K_{t} w_{t} w_{t}}^{t} \leq 0$, and (A4) $B_{K_{t}}^{t}, B_{w_{t}}^{t}$, and $B_{K_{t} w_{t}}^{t}$ are bounded for all $w_{t}$, where subscripts denote partial derivatives. (A2) and (A3) tell that higher $w_{t}$ raises the marginal benefit of development but at a decreasing rate. (A2) can be changed without affecting the results of the paper. (A3) provides a sufficient, but not necessary, condition for Theorem 1 which states that increased uncertainty reduces development. This assumption is widely used in analyzing the effects of uncertainty, as in Dixit and Pindyck (1994) and Pindyck (1988) where

\footnotetext{
${ }^{5}$ Choosing total capacity, instead of total capacity and current period investment level, as an argument for $B^{t}(\cdot)$ is for the sake of simplicity. In principle, the net benefit function should have three arguments: $B^{t}\left(K_{t}, I_{t}, w_{t}\right)=$ $R^{t}\left(K_{t}, w_{t}\right)-C^{t}\left(I_{t}, K_{t-1}\right)$, where $R^{t}(\cdot)$ and $C^{t}(\cdot)$ are revenue and cost functions respectively. A simplified special case of the cost function is $C^{t}\left(I_{t}+K_{t-1}\right)$. The implicit assumption of this simplification is that the cost of building $K_{t-1}$ is divided over all periods, so that even if $I_{t}=0$, there is still cost of constructing $K_{t-1}$ appropriated to period $t$. This cost function $C\left(K_{t}\right)$ leads to the specification of $B\left(K_{t}, w_{t}\right)$. This benefit function makes $I_{1}$ and $I_{2}$ perfect substitutes in case of perfect reversibility, which helps highlight the role of costly restoration and makes the analysis much easier without changing the nature of the problem. Viscusi (1985) made a similar assumption.
} 


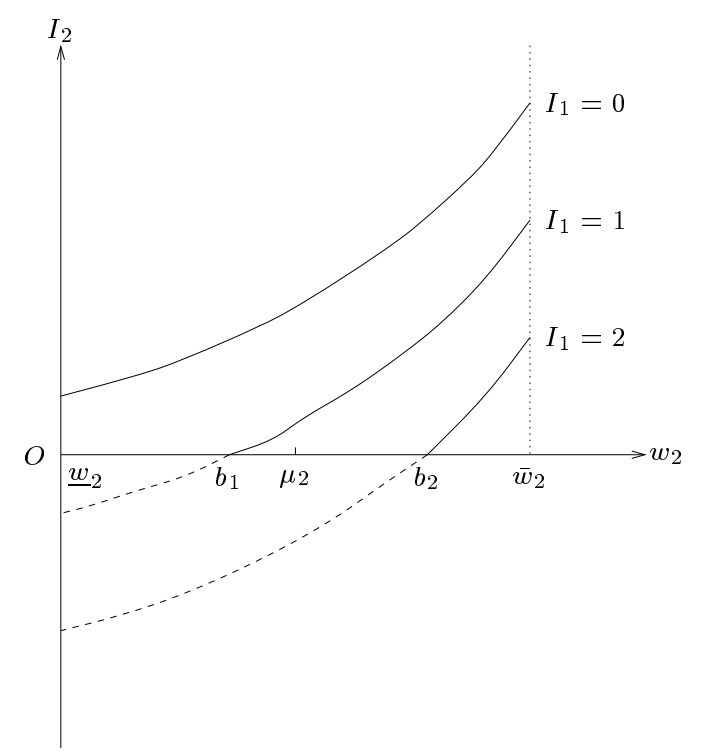

Figure 1: Optimal $I_{2}$ depending on $\varepsilon_{2}$ and $I_{1}$

$B_{K_{t} w_{t} w_{t}}^{t}=0$ is assumed. ${ }^{6}$ (A4) gives some regularity conditions.

Consider two period sequential resource development with complete resolution of uncertainty (that is, the value of the random variable is observed at the beginning of each period). The decision maker's optimization problem is:

$$
\begin{gathered}
\max _{I_{1}} B^{1}\left(I_{1}\right)+E_{w_{2}} \max _{I_{2}} B^{2}\left(I_{1}+I_{2}, w_{2}\right) \\
\text { s.t. } \quad I_{t} \geq 0, t=1,2 ; \quad w_{2}=\mu_{2}+\varepsilon_{2} ; \quad \varepsilon_{2} \sim\left(0, \sigma^{2}\right) ; \quad w_{2} \in\left[\underline{w}_{2}, \bar{w}_{2}\right]
\end{gathered}
$$

where $\sigma^{2}$ represents the level of uncertainty, $\underline{w}_{2}$ may be $-\infty$ and $\bar{w}_{2}$ may be $\infty$. For simplicity, we ignored $w_{1}$ in the $B^{1}$ function.

Appendix A derives the optimal second period development, $\hat{I}_{2}$, depending on $I_{1}$ and $w_{2}$, illustrated in Figure 1. The dashed curves indicate the optimal levels of $I_{2}$ when the investment is perfectly reversible: $I_{1}$ is so high and $w_{2}$ is so low that the optimal strategy is to restore. However, due to irreversibility $\hat{I}_{2}=0$ in this case.

For any $I_{1}$ (e.g. $I_{1}=2$ in Figure 1 ), there is a critical level of $w_{2}$, denoted as $w_{2}^{c}\left(I_{1}\right)$ (e.g. $b_{2}$

\footnotetext{
${ }^{6}$ In a static framework, $B_{K_{t} w_{t} w_{t}}^{t}<0$ means that increased uncertainty in $w_{t}$ reduces expected optimal $K_{t}$. We will show that, as in ROT, dynamic optimization further enhances this effect of uncertainty. (A3) guarantees that the static and dynamic effects of uncertainty work in the same direction.
} 
in Figure 1), below which the irreversibility constraint is binding (formal definition in Appendix A). When it is binding, there is a loss in the second period compared with the scenario of perfect reversibility (e.g. optimization without the constraint $I_{2} \geq 0$ ). We define irreversibility cost to be the expected loss in the second period associated with $I_{1}$ :

$$
I C\left(I_{1}\right)=\int_{\underline{w}_{2}}^{w_{2}^{c}\left(I_{1}\right)}\left(B^{2}\left(I_{1}+I_{2}^{r}\left(I_{1}, x\right), x\right)-B^{2}\left(I_{1}, x\right)\right) f(x) d x,
$$

where $f(\cdot)$ is the density function of $w_{2}$, and $I_{2}^{r}\left(I_{1}, w_{2}\right)$ is the optimal second period development without the irreversibility constraint, that is,

$$
I_{2}^{r}\left(I_{1}, w_{2}\right)=\arg \max _{I_{2}} B^{2}\left(I_{1}+I_{2}, w_{2}\right) .
$$

Theorem 4 in Appendix A shows that $I C\left(I_{1}\right)$ is increasing and convex in $I_{1}$.

The maximization problem in (1) can be transformed to directly include the irreversibility cost in the objective function. The maximization is equivalent to a situation where the decision maker pays the irreversibility cost in return for a perfectly reversible investment environment. Let

$$
M^{r}=E_{w_{2}} \max _{I_{2}} B^{2}\left(I_{1}+I_{2}, w_{2}\right)
$$

be the expected second period benefit with perfect reversibility. Then $E_{w_{2}} J\left(I_{1}, w_{2}\right)=M^{r}-I C\left(I_{1}\right)$ (Theorem 5 of Appendix A) and the maximization problem in (1) can be rewritten as:

$$
\max _{I_{1} \geq 0} B^{1}\left(I_{1}\right)+M^{r}-I C\left(I_{1}\right)
$$

Note that $M^{r}$ is independent of $I_{1}$, because for any $I_{1}$, the optimal second period capacity $K_{2}^{*}$ is always achieved by adjusting the level of $I_{2}$. This is due to the perfect substitutability between $I_{1}$ and $I_{2}$ in the net benefit function. Thus the irreversibility cost fully captures $I_{1}$ 's impact on the second period decision. The presentation in (5) enables us to evaluate the impact of uncertainty on investment through its impact on the irreversibility cost:

Theorem 1 Let $F_{i}(\cdot), i=a, b$ be two cumulative distribution functions of $w_{2}$, with $F_{a}$ second order 
stochastically dominating $F_{b}$. The marginal irreversibility cost associated with $F_{a}$ is smaller than that with $F_{b}$. That is, the first period investment under $F_{a}$ is higher than under $F_{b}$.

A direct implication of Theorem 1 is that increased future uncertainty reduces optimal $I_{1}$, which is similar to Pindyck (1988). In his interpretation, higher uncertainty raises the "option value" of delaying partial investment, leading to a smaller project size. Here, increased uncertainty raises the probability of the irreversibility constraint being binding (regretting the original investment), prompting more cautious investment decisions.

The effects of uncertainty on the value of the project can be analyzed using (4) and (5). It depends on two effects, through $I C$ and through $M^{r}$ : As $\sigma^{2}$ increases, the irreversibility cost $(I C)$ increases since higher uncertainty raises the probability that the project is over-sized. The unconstrained second period benefit $M^{r}$ may or may not increase depending on the curvature of the benefit function $B^{2}\left(K_{2}, w_{2}\right)$. We can show that when $B^{2}\left(K_{2}, w_{2}\right)$ is linear in $w_{2}$, the increase in $M^{r}$ dominates that in $I C$, and the project value is increasing in uncertainty, which is consistent with Pindyck (1988). ${ }^{7}$ However, in resource development projects, the most significant uncertainty is of environmental impacts. In most cases, the loss associated with serious environmental damage is more than proportional the gain associated with environmental improvement. The benefit function may be concave in $w_{2}$, especially where the irreversibility constraint is binding. In this case, the increase in $I C$ due to higher uncertainty may dominate the increase in $M^{r}$, and the project value may be concave in the level of uncertainty.

It becomes clear then that the risk-neutral decision maker may demonstrate risk averse behavior: increased uncertainty may lead to both less development and less payoff. In this context, the irreversibility cost may be considered as an insurance premium: the decision maker pays $I C\left(I_{1}\right)$ in exchange for the insurance of perfect reversibility.

\footnotetext{
${ }^{7}$ Pindyck studied price uncertainty where per period payoff function is linear in the random variable.
} 
The potential future loss from current investment changes the direction of the impact of interest rate on resource allocation. Thus far, we have not considered discounting the second period explicitly, even though we allow $B^{1}$ and $B^{2}$ to be different. Assuming $B^{2}=\frac{1}{1+i} B^{2^{\prime}}\left(K_{2}, w_{2}\right)$ where $i$ is the discount rate and $B^{2^{\prime}}$ is the temporal benefit of the project, one can show that higher $i$ leads to higher $I_{1}$ (Theorem 6 in Appendix A). That is, higher discount rate leads to more, instead of less, initial development. Higher $i$ reduces second period benefit, but also reduces the irreversibility cost IC and MIC. Because of irreversibility, investment in first period has a much bigger impact on future cost than future benefit. Discounting thus reduces the net cost incurred in the future due to current development, leading to more current development.

This result is contrary to the conventional wisdom that discounting discourages investment. The inter-temporal pattern of cost and benefit in the conventional investment model is that cost occurs first and benefits later. But many natural resource projects have the opposite pattern: benefits are initially enjoyed at the cost of the future. Once the timing sequence of cost and benefit is reversed, the impact of discount rate on investment is reversed. Then with a higher discount rate, it is more likely that a big project is built now and restoration is conducted later.

Irreversibility cost is monotonically related to the much used concept in ROT, the ArrowFisher-Henry option value (OV). In fact, OV provides the lower bound for IC and equals IC for small projects. ${ }^{8}$ To see this, we modify our model following the investment problem in Arrow and Fisher (1974), where the decision of executing a project of size $K$ is made in either the current or the next period. Absolute irreversibility means that if the project is executed in period 1, the capacity remains at $K$ in both periods. Perfect reversibility means that the project can be reversed in the second period so that the capacity can be zero, depending on the state of nature. Then

\footnotetext{
${ }^{8}$ Since OV is the value of information conditional on the project being delayed (see Hanemann (1989)), our statement about the relationship between IC and OV also applies to that between IC and the (conditional) value of information.
} 
Theorem 2 The relation between $I C$ and $O V$ can be expressed as

$$
O V(K)=I C(K)-\max \left\{0, \int_{\underline{w}_{2}}^{\bar{w}_{2}} B^{2}\left(0, w_{2}\right) d F\left(w_{2}\right)-\int_{\underline{w}_{2}}^{\bar{w}_{2}} B^{2}\left(K, w_{2}\right) d F\left(w_{2}\right)\right\}
$$

where the second term on the right hand side is the expected gain of delaying the project calculated using the open-loop approach. Note that $O V(K)=I C(K)$ when $K$ is small, since the difference between the integrals in (6) is negative when $K$ is small and positive when $K$ is large.

To see the intuition of (6), note that in closed-loop optimization, if the project is executed, the decision maker gains by the extra services provided by the project in period one, $B^{1}(K)-B^{1}(0)$, and loses by the possibility that the project is too big (i.e. $I C(K)$ ). Thus, the net benefit of not delaying is $B^{1}(K)-B^{1}(0)-I C(K)$, and $I C(K)$ measures the benefit of delaying the project in the closed-loop approach. Similarly, the difference between the integrals in (6) measures the benefit of delaying in the open-loop approach. Since option value is in fact a correction factor imposed on the open-loop objective function. (6) follows from the fact that the benefit of executing the project is the same $\left(B^{1}(K)-B^{1}(0)\right)$ in both open and closed-loop approaches.

\section{Restoration and endogenous degree of irreversibility}

Now suppose that restoration is possible in the second period at a certain cost. Let $I_{R}$ be the amount of restoration (i.e. it is $-I_{2}$ when $I_{2}$ is negative), and $c_{0}$ and $c\left(I_{R}\right)$ the fixed and variable costs. Restoration efforts usually incur physical costs and transaction costs that are independent of restoration level. We assume that $c(\cdot)$ is convex with $c(0)=0$.

The new decision problem is:

$$
\begin{aligned}
\max _{I_{1}}\left\{B^{1}\left(I_{1}\right)+\int_{w_{2}^{c}\left(I_{1}\right)}^{\bar{w}_{2}} B^{2}\left(I_{1}\right.\right. & \left.+I_{2}^{r}\left(I_{1}, w_{2}\right), w_{2}\right) f\left(w_{2}\right) d w_{2} \\
& \left.+\int_{\underline{w}_{2}}^{w_{2}^{c}\left(I_{1}\right)}\left[\max _{I_{R}}\left(B^{2}\left(I_{1}-I_{R}, w_{2}\right)-c\left(I_{R}\right)-c_{0} s\left(I_{R}\right)\right)\right] f\left(w_{2}\right) d w_{2}\right\}
\end{aligned}
$$

where $s\left(I_{R}\right)=(>) 0$ if $I_{R}=(>) 0$. The second component in (7) is the expected second period 


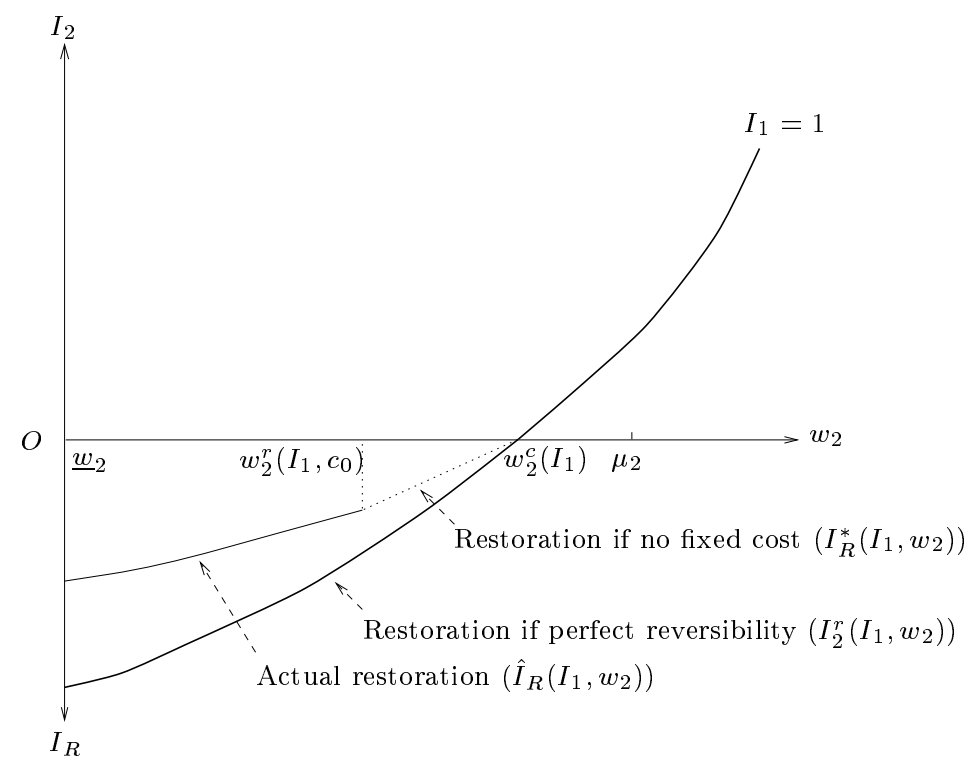

Figure 2: Restoration given $I_{1}$

benefit when further development is needed (so $I_{2}^{r}$ is used). The third component is the expected benefit when restoration is needed and its amount has to be optimally chosen.

The optimal restoration decisions are illustrated in Figure 2. At $w_{2}=w_{2}^{c}\left(I_{1}\right)$, there is no incentive for restoration. As $w_{2}$ decreases, restoration is not feasible at first, since the variable benefit of restoration is small and cannot compensate for the fixed cost that would have to be incurred if restoration is undertaken. However, as $w_{2}$ further declines, the variable benefit of restoration increases, and eventually will surpass the fixed cost when $w_{2}$ falls below a critical level, denoted as $w_{2}^{r}\left(I_{1}, c_{0}\right)$, and restoration occurs. We denote this optimal restoration level as $I_{R}^{*}\left(I_{1}, w_{2}\right)$. It is straightforward to show that (see Appendix B) the critical level $w_{2}^{r}\left(I_{1}, c_{0}\right)$ is increasing in $I_{1}$ and decreasing in $c_{0}$.

Using the irreversibility cost approach of the last section, (7) can be rewritten as:

$$
\max _{I_{1}} B^{1}\left(I_{1}, w_{1}\right)+M^{r}-I C_{R}\left(I_{1}, c_{0}\right)
$$

where $M^{r}$, defined in (4), is the second period benefit with free restoration (or perfect reversibility), and $I C_{R}\left(I_{1}, c_{0}\right)$ (defined in Appendix B) is the irreversibility cost with restoration, an extension 
of irreversibility cost $I R . I C_{R}$ is increasing, although not necessarily convex, in $I_{1}$. But the concavity of $B^{1}(\cdot)$ and $B^{2}(\cdot)$ and convexity of $c(\cdot)$ ensure that a unique solution exists in (8). More importantly,

Theorem 3 The marginal irreversibility cost with restoration, $M I C_{R}\left(I_{1}, c_{0}\right)$, is increasing in $c_{0}$. Specifically,

$$
M I C_{R}\left(I_{1}, c_{0}\right)=\int_{w_{2}^{r}\left(I_{1}, c_{0}\right)}^{w_{2}^{c}\left(I_{1}\right)}-\frac{\partial B^{2}\left(I_{1}, w_{2}\right)}{\partial I_{1}} f\left(w_{2}\right) d w_{2}+\int_{\underline{w}_{2}}^{w_{2}^{r}\left(I_{1}, c_{0}\right)} c^{\prime}\left(I_{R}^{*}\left(I_{1}, w_{2}\right)\right) f\left(w_{2}\right) d w_{2}
$$

From (9), we see that $M I C_{R}$ is increasing in the marginal restoration cost $c^{\prime}(\cdot)$. Fixed cost $c_{0}$ affects $M I C_{R}\left(I_{1}, c_{0}\right)$ through $w_{2}^{r}\left(I_{1}, c_{0}\right)$, or by affecting the range of $w_{2}$ where restoration is economically feasible. Since higher $c_{0}$ reduces optimal first period development $\hat{I}_{1}$, and as Appendix B shows, $\hat{I}_{R}\left(I_{1}, w_{2}\right)$ is increasing in $\hat{I}_{1}$, we know

$$
\frac{\partial \hat{I}_{R}\left(\hat{I}_{1}\left(c_{0}\right), w_{2}\right)}{\partial c_{0}} \leq 0 ; \quad E_{w_{2}} \frac{\partial \hat{I}_{R}\left(\hat{I}_{1}\left(c_{0}\right), w_{2}\right)}{\partial c_{0}} \leq 0
$$

Higher fixed cost of restoration reduces the expected restoration and the restoration effort for each state of nature. The intuition is that as the fixed restoration cost increases, the decision maker would prefer a lower probability of restoration, leading to less development in the first period. Thus less restoration is optimal for each state of nature. We can then conclude that

Remark 1 In a dynamic and stochastic framework, the fixed cost of restoration affects not only whether or not there is restoration but also the level of restoration.

In fact, we can use our framework to show that the marginal value of investment, i.e. the marginal $q$ in the neoclassical investment theory (Abel and Eberly (1994)), depends continuously on $c_{0}$. For example, by (8) we can deduct that the value function of second period investment is $V_{2}\left(I_{1}, c_{0}\right)=M^{r}-I C_{R}\left(I_{1}, c_{0}\right)$. Then clearly the marginal value of a size $I_{1}$ project (which is negative in our special case) is decreasing in $c_{0}$. 
The discussion so far indicates that in the second period restoration may be needed. The probability and magnitude of restoration depend on the downside support of the random variable $\underline{w}_{2}$, the fixed cost $c_{0}$, and initial development $I_{1}$. For a given $I_{1}$, absolute irreversibility corresponds to a situation where the fixed cost of restoration is so high that the probability of restoration is zero, or $w_{2}^{r}\left(I_{1}, c_{0}\right)=\underline{w}_{2}$. It does not depend on the marginal cost of restoration, since a higher marginal cost would only lead to a smaller amount of restoration (but does not lead to no restoration), unless the marginal cost is infinite. Caballero (1991) identified absolute irreversibility with infinite marginal restoration cost. Then absolute irreversibility is a rare event since infinite marginal cost rarely happens. However, we show here that absolute irreversibility happens as long as the fixed cost of negative adjustment is reasonably high. This scenario is much more likely to happen.

This interpretation means that irreversibility is a dynamic concept and evolves with changes in parameters. For example, changes in technologies that reduce $c_{0}$ and changes in tastes that increase the damage of development may make irreversible projects in the past become "restorable" now.

The degree of irreversibility is endogenously determined when $I_{1}$ is chosen. Absolute irreversibility is less likely for large projects than for small projects. ROT assumes away this endogeneity by assuming absolute irreversibility. Therefore, allowing variable project size in ROT conflicts with the absolute irreversibility assumption.

The cost of assuming absolute irreversibility (and ignoring restoration possibilities) depends on the nature of the problem. Ceteris paribus, it is small if both the fixed and variable costs of restoration are high. If resource development leads to technical irreversibility, such as extinction of species, then the restoration cost is infinite and the assumption of absolute (economic) irreversibility is justified. ${ }^{9}$ In some cases such as urban sprawl when agricultural or forest lands are converted to

\footnotetext{
${ }^{9}$ Even in this case, we should be careful when asserting absolute irreversibility. If a species exists in different parts of the world, then extinction in one area is not irreversible: with some cost, the same species can be reintroduced in this area. In another case, if there are close substitutes to a species (either in terms of their gene structure or in
} 
urban use, the cost of restoration is high and the assumption of irreversibility is realistic. However, in other cases such as restoring the fisheries that are damaged by water development projects, the restoration cost is sufficiently low so that the assumption of absolute irreversibility is unrealistic. In fact, we have witnessed significant restoration efforts in fisheries. ${ }^{10}$

\section{Discussion and Conclusion}

This paper expands ROT to situations where a natural resource development project may make the decision maker worse-off and costly restoration is feasible. We introduced a notion of irreversibility cost which is the expected cost due to the irreversibility constraint and restoration cost. This concept is related to, but more general than the Arrow-Fisher-Henry option value.

Even without relaxing the assumption of absolute irreversibility, the implications of ROT may be different for resource projects than those for conventional investment projects. We showed that the value of a project may not be convex in the underlying random variable, due to the possibility of benefit loss of an over-sized project. A risk neutral decision maker may thus demonstrate risk averse behavior: both the scale of development and the associated payoff go down as the level of uncertainty rises. This result highlights the importance of information gathering and uncertainty resolution in project formulation.

We also showed that for resource projects, higher discount rate may in fact lead to more development. It encourages the mentality of "building now and restoring later." This result has important implications for the choice of discount rate in resource project evaluation. Low discount rate has been blamed for excessive resource development. We argue that this is mainly due to the practice of neglecting the possibility of costly restoration in formulating and evaluating resource

terms of consumption substitution), its extinction is likely to incur economic losses (i.e. we wish to restore it) only in very extreme situations. In this case, irreversibility can be safely ignored: there may be absolute irreversibility, but essentially it does not affect the optimal resource development.

${ }^{10}$ One example is the Central Valley Project Improvement Act (CVPIA) in California which allocates water to help restore salmon fishery in San Francisco Bay Delta that was negatively impacted by water development. 
projects. Once costly restoration is considered, the reverse may be true: low discount rate may encourage more cautious development decisions.

When costly restoration is allowed, the degree of irreversibility becomes an endogenous choice. Absolute irreversibility happens only when the fixed restoration cost is sufficiently high and when the random variable has a sufficiently high downside support (i.e. a bad scenario cannot happen). ROT, by assuming absolute irreversibility, assumes away an endogenous choice. Further, contrary to the popular argument that large projects tend to be irreversible, we find that absolute irreversibility is more likely for small projects.

We showed that understanding the structure of restoration cost function is important: the fixed cost of restoration affects not only whether or not restoration should be undertaken, but also the level of development (and restoration) decisions. More generally, the marginal value of investment, i.e. the marginal $q$ in neoclassical investment theory, continuously depends on the fixed cost.

Although we have focused on resource development projects, the framework can be used to analyze more conventional investment decisions where costly correction of mistakes is allowed. In essence, restoration is a way of correcting the mistake of too much initial investment. Similar scenario may happen in other settings, such as a certain investment leading to the possibility of environmental liability. One example is the federal Superfund cleanup program, showing that a profitable investment may bring forth an environmental liability that out-weight the earned profit (Acton (1989)). 


\section{Appendix A: Details of the Basic Model}

We denote the second period maximization problem in (1) as $J\left(I_{1}, w_{2}\right)=\max _{I_{2} \geq 0} B^{2}\left(I_{1}+I_{2}, w_{2}\right)$ and its solution as $\hat{I}_{2}\left(I_{1}, w_{2}\right)$. For $\hat{I}_{2}\left(I_{1}, w_{2}\right)>0$, it is easy to verify that $\frac{\partial \hat{I}_{2}\left(I_{1}, w_{2}\right)}{\partial w_{2}}>0$ and $\frac{\partial \hat{I}_{2}\left(I_{1}, w_{2}\right)}{\partial I_{1}}<0$. This result leads to Figure 1.

The formal definition of $w_{2}^{c}\left(I_{1}\right)$ is:

Definition 1 For a given $I_{1}$, the associated critical $w_{2}$ is defined as

$$
w_{2}^{c}\left(I_{1}\right)=\sup \left\{w_{2} \in\left[\underline{w}_{2}, \bar{w}_{2}\right]: \hat{I}_{2}\left(I_{1}, w_{2}\right)=0\right\} \bigcup \inf \left\{w_{2} \in\left[\underline{w}_{2}, \bar{w}_{2}\right]: \hat{I}_{2}\left(I_{1}, w_{2}\right)>0\right\}
$$

Theorem 4 The irreversibility cost is increasing and convex in $I_{1}$.

Proof: From the definition of threshold $w_{2}$ level, we know $I_{2}^{r}\left(I_{1}, w_{2}^{c}\left(I_{1}\right)\right)=0$. The first order condition of (3) gives $\frac{\partial B^{2}\left(I_{1}+I_{2}^{r}, w_{2}\right)}{\partial I_{2}}=0$. We then have

$$
\begin{aligned}
M I C=\frac{\partial I C}{\partial I_{1}} & =\left(B^{2}\left(I_{1}+I_{2}^{r}\left(I_{1}, w_{2}^{c}\left(I_{1}\right)\right), w_{2}^{c}\left(I_{1}\right)\right)-B^{2}\left(I_{1}, w_{2}^{c}\left(I_{1}\right)\right)\right) f\left(w_{2}^{c}\left(I_{1}\right)\right) \frac{\partial w_{2}^{c}\left(I_{1}\right)}{\partial I_{1}} \\
& +\int_{\underline{w}_{2}}^{w_{2}^{c}\left(I_{1}\right)}\left(\frac{\partial B^{2}\left(I_{1}+I_{2}^{r}, x\right)}{\partial I_{2}}\left(1+\frac{\partial I_{2}^{r}}{\partial I_{1}}\right)-\frac{\partial B^{2}\left(I_{1}, x\right)}{\partial I_{1}}\right) f(x) d x \\
& =0+\int_{\underline{w}_{2}}^{w_{2}^{c}\left(I_{1}\right)}\left(0 *\left(1+\frac{\partial I_{2}^{r}}{\partial I_{1}}\right)-\frac{\partial B^{2}\left(I_{1}, x\right)}{\partial I_{1}}\right) f(x) d x \\
& =\int_{\underline{w}_{2}}^{w_{2}^{c}\left(I_{1}\right)}-\frac{\partial B^{2}\left(I_{1}, x\right)}{\partial I_{1}} f(x) d x
\end{aligned}
$$

For any $w_{2}<w_{2}^{c}\left(I_{1}\right), \frac{\partial B^{2}\left(I_{1}, w_{2}\right)}{\partial I_{1}}<\frac{\partial B^{2}\left(I_{1}+I_{2}^{r}, w_{2}\right)}{\partial I_{1}}$ since $I_{2}^{r}\left(I_{1}, w_{2}\right)<0$ and $B^{2}(\cdot)$ is concave in $K$. But $\frac{\partial B^{2}\left(I_{1}+I_{2}^{r}, w_{2}\right)}{\partial I_{1}}=\frac{\partial B^{2}\left(I_{1}+I_{2}^{r}, w_{2}\right)}{\partial I_{2}}=0$. Therefore, for any $w_{2}<w_{2}^{c}\left(I_{1}\right), \frac{\partial B^{2}\left(I_{1}, w_{2}\right)}{\partial I_{1}}<0$. Substituting this result into $(12)$, we get $M I C^{\partial I_{2}}>0$.

Similarly, differentiating Equation 12 with respect to $I_{1}$, we have $\frac{\partial M I C\left(I_{1}, w_{1}\right)}{\partial I_{1}}=0+\int_{\underline{w}_{2}}^{w_{2}^{c}\left(I_{1}\right)}-\frac{\partial^{2} B^{2}\left(I_{1}\right)}{\partial I_{1}^{2}} f(x) d x$. Since $B^{2}(\cdot)$ is concave in $I_{1}, \frac{\partial M I C\left(I_{1}, w_{1}\right)}{\partial I_{1}}>0$.

Theorem 5 For any $I_{1}, E_{w_{2}} J\left(I_{1}, w_{2}\right)=M^{r}-I C\left(I_{1}\right)$.

\section{Proof:}

$$
\begin{aligned}
E_{w_{2}} J\left(I_{1}, w_{2}\right) & =\int_{\underline{w}_{2}}^{\bar{w}_{2}} \max _{I_{2} \geq 0} B^{2}\left(I_{1}+I_{2}, x\right) f(x) d x \\
& =\int_{\underline{w}_{2}}^{w_{2}^{c}\left(I_{1}\right)} B^{2}\left(I_{1}, x\right) f(x) d x+\int_{w_{2}^{c}\left(I_{1}\right)}^{\bar{w}_{2}} B^{2}\left(I_{1}+\hat{I}_{2}, x\right) f(x) d x \\
M^{r} & =\int_{\underline{w}_{2}}^{\bar{w}_{2}} \max _{I_{2}} B^{2}\left(I_{1}+I_{2}, x\right) f(x) d x \\
& =\int_{\underline{w}_{2}}^{\bar{w}_{2}} B^{2}\left(I_{1}+I_{2}^{r}, x\right) f(x) d x \\
& =\int_{\underline{w}_{2}}^{w_{2}^{c}\left(I_{1}\right)} B^{2}\left(I_{1}+I_{2}^{r}, x\right) f(x) d x+\int_{w_{2}^{c}\left(I_{1}\right)}^{\bar{w}_{2}} B^{2}\left(I_{1}+I_{2}^{r}, x\right) f(x) d x
\end{aligned}
$$


Subtracting (13) from (14), and noting that for $w_{2} \geq w_{2}^{c}\left(I_{1}\right), I_{2}^{r}=\hat{I}_{2}$,

$$
\begin{aligned}
M^{r}-E_{w_{2}} J\left(I_{1}, w_{2}\right) & =\int_{\underline{w}_{2}}^{w_{2}^{c}\left(I_{1}\right)}\left(B^{2}\left(I_{1}+I_{2}^{r}, x\right)-B^{2}\left(I_{1}, x\right)\right) f(x) d x \\
& =I C\left(I_{1}\right)
\end{aligned}
$$

Theorem 6 Optimal $I_{1}$ increases as discount rate $i$ increases.

Proof: Note that $I C\left(I_{1}\right)=\frac{1}{1+i} I C^{\prime}\left(I_{1}\right)$ where $I C^{\prime}\left(I_{1}\right)$ is given in (2) with $B^{2}$ replaced by $B^{2^{\prime}}$. Then the theorem follows immediately from (5).

\section{Appendix B: Details of the Model with Endogenous Irreversibility}

We first deal with the third component of $(7)$, that is, the restoration problem given $I_{1}$ and $w_{2}<w_{2}^{c}\left(I_{1}\right)$. Denote the restoration payoff associated with a certain $I_{1}$ and $w_{2}$ as

$$
V\left(I_{1}, w_{2}, I_{R}\right)=B^{2}\left(I_{1}-I_{R}, w_{2}\right)-c\left(I_{R}\right)-c_{0} s\left(I_{R}\right)
$$

and let $\hat{I}_{R}\left(I_{1}, w_{2}\right)=\arg \max _{I_{R}} V\left(I_{1}, w_{2}, I_{R}\right)$.

To understand the behavior of $\hat{I}_{R}\left(I_{1}, w_{2}\right)$, let $I_{R}^{*}\left(I_{1}, w_{2}\right)$ be the optimal restoration without the fixed cost, i.e. it solves $\partial V / \partial I_{R}=0$. Then there is positive amount of restoration with $\hat{I}_{R}\left(I_{1}, w_{2}\right)=I_{R}^{*}\left(I_{1}, w_{2}\right)$ only if the variable benefit of restoration surpasses the fixed cost. This result happens only when $w_{2}$ is below a critical level, denoted as $w_{2}^{r}\left(I_{1}, c_{0}\right)$.

Definition 2 The critical level of $w_{2}$ for restoration, $w_{2}^{r}\left(I_{1}, c_{0}\right)$, is defined as

$$
w_{2}^{r}\left(I_{1}, c_{0}\right)=\sup \left\{w_{2} \in\left[\underline{w}_{2}, w_{2}^{c}\left(I_{1}\right)\right]: \hat{I}_{R}\left(I_{1}, w_{2}\right)>0\right\} \bigcup \inf \left\{w_{2} \in\left[\underline{w}_{2}, w_{2}^{c}\left(I_{1}\right)\right]: \hat{I}_{R}\left(I_{1}, w_{2}\right)=0\right\}
$$

It is obvious that

$$
\hat{I}_{R}\left(I_{1}, w_{2}\right) \begin{cases}=I_{R}^{*}\left(I_{1}, w_{2}\right) & \text { if } w_{2}<w_{2}^{r}\left(I_{1}, c_{0}\right) \\ =0 & \text { if } w_{2} \geq w_{2}^{r}\left(I_{1}, c_{0}\right)\end{cases}
$$

It is straightforward to show that

$$
\frac{\partial w_{2}^{r}\left(I_{1}, c_{0}\right)}{\partial I_{1}} \geq 0 ; \quad \frac{\partial w_{2}^{r}\left(I_{1}, c_{0}\right)}{\partial c_{0}} \leq 0 ; \quad \frac{\partial \hat{I}_{R}\left(I_{1}, w_{2}\right)}{\partial I_{1}} \geq 0 ; \quad \frac{\partial \hat{I}_{R}\left(I_{1}, w_{2}\right)}{\partial w_{2}} \leq 0
$$

Higher initial development or lower fixed restoration cost increases $w_{2}^{r}\left(I_{1}, c_{0}\right)$ and the probability that restoration is optimal. The amount of restoration increases with the initial investment and declines with $w_{2}$.

To incorporate the restoration consideration in the optimal choice of $I_{1}$, substitute the solution $\hat{I}_{R}\left(I_{1}, w_{2}\right)$ into (7). Then the maximization problem becomes:

$$
\begin{aligned}
& \max _{I_{1}} B^{1}\left(I_{1}, w_{1}\right)+\int_{w_{2}^{c}\left(I_{1}\right)}^{\bar{w}_{2}} \overbrace{B^{2}\left(I_{1}+I_{2}^{r}\left(I_{1}, w_{2}\right), w_{2}\right) f\left(w_{2}\right) d w_{2}}^{\text {adding capacity }}+\int_{w_{2}^{r}\left(I_{1}, c_{0}\right)}^{w_{2}^{c}\left(I_{1}\right)} \overbrace{B^{2}\left(I_{1}, w_{2}\right) f\left(w_{2}\right) d w_{2}}^{\text {no action }} \\
& +\int_{\underline{w}_{2}}^{w_{2}^{r}\left(I_{1}, c_{0}\right)} \underbrace{\left(B^{2}\left(I_{1}-I_{R}^{*}\left(I_{1}, w_{2}\right), w_{2}\right)-c\left(I_{R}^{*}\left(I_{1}, w_{2}\right)\right)-c_{0}\right) f\left(w_{2}\right) d w_{2}}_{\text {restoration }}
\end{aligned}
$$

Using the definition of $M^{r},(20)$ can be rewritten as (8), and the expected loss in period two due to 
costly restoration is

$$
\begin{gathered}
I C_{R}\left(I_{1}, c_{0}\right)=\int_{w_{2}^{r}\left(I_{1}, c_{0}\right)}^{w_{2}^{c}\left(I_{1}\right)} \overbrace{\left[B^{2}\left(I_{1}+I_{2}^{r}\left(I_{1}, w_{2}\right), w_{2}\right)-B^{2}\left(I_{1}, w_{2}\right)\right] f\left(w_{2}\right) d w_{2}}^{\text {loss due to absolute irreversibility }}+ \\
\int_{\underline{w}_{2}}^{w_{2}^{r}\left(I_{1}, c_{0}\right)} \underbrace{\left[B^{2}\left(I_{1}+I_{2}^{r}\left(I_{1}, w_{2}\right), w_{2}\right)-B^{2}\left(I_{1}-I_{R}^{*}\left(I_{1}, w_{2}\right), w_{2}\right)+c\left(I_{R}^{*}\left(I_{1}, w_{2}\right)\right)+c_{0}\right] f\left(w_{2}\right) d w_{2}}_{\text {loss due to costly restoration }}
\end{gathered}
$$

The first term on the right hand side of (21) is the cost of absolute irreversibility (when restoration is not optimal), and the second term is the sum of the cost of restoration and irreversibility (when the restoration is partial). Note that if $c_{0}$ is infinity, $w_{2}^{r}\left(I_{1}, c_{0}\right)=\underline{w}_{2}$ and we are left with only the first term, while if $c_{0}$ is zero, $w_{2}^{r}\left(I_{1}, c_{0}\right)=w_{2}^{c}\left(I_{1}\right)$ and we have only the second term.

\section{Appendix C: Proof of Theorems}

Theorem 1. Let $h\left(w_{2}\right)=-\frac{\partial B^{2}\left(I_{1}, w_{2}\right)}{\partial I_{1}}$. We know $h\left(w_{2}\right)>0$ for $w_{2}<w_{2}^{c}\left(I_{1}\right)$ and $h\left(w_{2}^{c}\left(I_{1}\right)\right)=0$. Further, from assumptions (A2)-(A4), we know $h^{\prime}\left(w_{2}\right)<0, h^{\prime \prime}\left(w_{2}\right) \geq 0$, and $h\left(w_{2}\right)$ and $h^{\prime}\left(w_{2}\right)$ are bounded for all $w_{2}$. Using these conditions and integrating by parts, we can show that $M I C_{a}-M I C_{b}=$ $-h^{\prime}\left(w_{2}^{c}\left(I_{1}\right)\right) \int_{\underline{w}_{2}}^{w_{2}^{c}\left(I_{1}\right)}\left(F_{a}(y)-F_{b}(y)\right) d y+\int_{\underline{w}_{2}}^{w_{2}^{c}\left(I_{1}\right)} \int_{\underline{w}_{2}}^{x}\left(F_{a}(y)-F_{b}(y)\right) d y h^{\prime \prime}(x) d x<0$.

Theorem 2 With the new formulation, the critical $w_{2}$ level below which the irreversibility constraint is binding becomes $w_{2}^{c}(K)$ and the irreversibility cost is

$$
I C(K)=\int_{\underline{w}_{2}}^{w_{2}^{c}(K)}\left(B^{2}\left(0, w_{2}\right)-B^{2}\left(K, w_{2}\right)\right) d F\left(w_{2}\right) .
$$

The AFH option value is defined by $O V(K)=[\hat{V}(0)-\hat{V}(1)]-[\tilde{V}(0)-\tilde{V}(1)]$, where

$$
\hat{V}(0)=B^{1}(0)+\int_{\underline{w}_{2}}^{w_{2}^{c}(K)} B^{2}\left(0, w_{2}\right) d F\left(w_{2}\right)+\int_{w_{2}^{c}(K)}^{\bar{w}_{2}} B^{2}\left(K, w_{2}\right) d F\left(w_{2}\right)
$$

is the expected payoff of delaying the project in a closed-loop optimization,

$$
\hat{V}(1)=B^{1}(K)+\int_{\underline{w}_{2}}^{\bar{w}_{2}} B^{2}\left(K, w_{2}\right) d F\left(w_{2}\right)
$$

is the expected payoff of executing the project in a closed-loop optimization,

$$
\tilde{V}(0)=B^{1}(0)+\max \left\{\int_{\underline{w}_{2}}^{\bar{w}_{2}} B^{2}\left(K, w_{2}\right) d F\left(w_{2}\right), \int_{\underline{w}_{2}}^{\bar{w}_{2}} B^{2}\left(0, w_{2}\right) d F\left(w_{2}\right)\right\}
$$

is the payoff of delaying execution in the open-loop optimization, and

$$
\tilde{V}(1)=B^{1}(K)+\int_{\underline{w}_{2}}^{\bar{w}_{2}} B^{2}\left(K, w_{2}\right) d F\left(w_{2}\right)
$$

is the payoff of not delaying in the open-loop optimization. (6) then follows immediately.

Theorem 3. Similar to proving Theorem 4, by applying $(17), \frac{\partial B^{2}\left(I_{1}+I_{2}^{r}, w_{2}\right)}{\partial I_{1}}=0$, and $B_{1}^{2}\left(I_{1}-I_{R}^{*}, w_{2}\right)+$ 
$c^{\prime}\left(I_{R}^{*}\right)=0$, we can show

$$
M I C_{R}\left(I_{1}, c_{0}\right)=\int_{w_{2}^{r}\left(I_{1}, c_{0}\right)}^{w_{2}^{c}\left(I_{1}\right)}-B_{1}^{2}\left(I_{1}, w_{2}\right) f\left(w_{2}\right) d w_{2}+\int_{\underline{w}_{2}}^{w_{2}^{r}\left(I_{1}, c_{0}\right)}-B_{1}^{2}\left(I_{1}-I_{R}^{*}\left(I_{1}, w_{2}\right), w_{2}\right) f\left(w_{2}\right) d w_{2}
$$

which is equal to (9). Taking derivatives on (23), we can show:

$\frac{\partial M I C_{R}\left(I_{1}, c_{0}\right)}{\partial c_{0}}=\left(B_{1}^{2}\left(I_{1}, w_{2}^{r}\left(I_{1}, c_{0}\right)\right)-B_{1}^{2}\left(I_{1}-I_{R}^{*}\left(I_{1}, w_{2}^{r}\left(I_{1}, c_{0}\right)\right), w_{2}^{r}\left(I_{1}, c_{0}\right)\right)\right) f\left(w_{2}^{r}\left(I_{1}, c_{0}\right)\right) \frac{\partial w_{2}^{r}\left(I_{1}, c_{0}\right)}{\partial c_{0}}>0$. 


\section{References}

Abel, Andrew B. and Janice C. Eberly, "A Unified Model of Investment Under Uncertainty," American Economic Review, 1994, 84 (5), 1369-1384.

Acton, Jan Paul, "Understanding Superfund: A Progress Report," Technical Report RAND/R3838-ICJ, RAND 1989.

Arrow, Kenneth J. and Anthony C. Fisher, "Environmental Preservation, Uncertainty, and Irreversibility," Quarterly Journal of Economics, 1974, 88, 312-319.

Caballero, Ricardo J., "On the Sign of the Investment-Uncertainty Relationship," American Economic Review, 1991, 81 (1), 279-288.

Capozza, Dennis and Yuming Li, "The Intensity and Timing of Investment: The Case of Land," American Economic Review, September 1994, 84 (4), 889-904.

Dixit, Avinash K. and Robert S. Pindyck, Investment Under Uncertainty, Princeton, N.J.: Princeton University Press, 1994.

Epstein, Larry G., "Decision Making and the Temporal Resolution of Uncertainty," International Economic Review, 1980, 21, 269-283.

Fisher, Anthony C., John V. Krutilla, and Charles J. Cicchetti, "The Economics of Environmental Preservation: A Theoretical and Empirical Analysis," American Economic Review, 1972, 62, 605-619.

Freixas, Xavier and Jean-Jacques Laffont, "On the Irreversibility Effect," in Marcel Boyer and Richard Kihlstrom, eds., Bayesian Models in Economic Theory, North-Holland, 1984, chapter 7, pp. 105-114. 
Hanemann, W. Michael, "Information and the Concept of Option Value," Journal of Environmental Economics and Management, 1989, 16, 23-37.

Henry, Claude, "Investment Decisions Under Uncertainty: The Irreversibility Effect," American Economic Review, 1974, 64, 1006-1012.

Kolstad, Charles, "Fundamental Irreversibilities in Stock Externalities," Journal of Public Economics, 1996, 60(2), 221-233.

Majd, Saman and Robert Pindyck, "Time to Build, Option Value and Investment Decisions," Journal of Financial Economics, 1987, 18 (1), 7-27.

McDonald, R. and D. Siegel, "The Value of Waiting to Invest," Quarterly Journal of Economics, 1986, 101, 707-727.

Pindyck, Robert S., "Irreversibile Investment, Capacity Choice, and the Value of the Firm," American Economic Review, 1988, 79, 969-985.

Ulph, Alistair and David Ulph, "Global Warming, Irreversibility and Learning," Economic Journal, 1997, 107, 636-650.

Viscusi, W. Kip, "Environmental Policy Choice with an Uncertain Chance of Irreversibility," Journal of Environmental Economics and Management, March 1985, pp. 28-44. 